and QRISK2 and the degree of airflow obstruction (FEV1: $\mathrm{r}=-0.470^{* *}$, rho $\left.=-0.325^{*}\right)$, emphysema (KCO: $\mathrm{r}=-0.493^{* *}, \mathrm{r}=-0.411^{* *}$ ) and hyperinflation (RV\%TLC: $r=0.550 * *, r=0.433 * *)$. Including only patients with $\geq 3$ PFT results, we found a significant relationship between emphysema progression and calculated but not measured cardiovascular risk: KCO change vs. QRISK2 ( $\mathrm{r}=-0.549, \mathrm{p}=0.015, \mathrm{n}=19)$.

Conclusions Reduced lung function was associated with a greater magnitude of measured and calculated cardiac risk in the AATD cohort. However AATD-mediated lung disease progression was not significantly related to measured CV risk - an exception being a significant relationship between the rate of decline of KCO and QRISK2.

\section{P137 WHY IS ERDOSTEINE RECOMMENDED AS A TREATMENT FOR ACUTE EXACERBATIONS OF CHRONIC BRONCHITIS? A SYSTEMATIC REVIEW OF CLINICAL TRIALS}

${ }^{1} \mathrm{CL}$ Johnson, ${ }^{2} \mathrm{DF}$ Rogers. ${ }^{1}$ Imperial College London, London, UK; ${ }^{2}$ National Heart and Lung Institute, Imperial College London, London, UK

\subsection{6/thoraxjnl-2016-209333.280}

Background Exacerbations of chronic bronchitis or chronic obstructive pulmonary disease (COPD) are a significant health burden. A substantial component of these exacerbations in many patients is mucus-hypersecretion. Mucolytics, drugs that 'thin' mucus, have potential efficacy in these patients.

Currently, erdosteine is the only mucolytic in the British National Formulary (BNF) indicated for "symptomatic treatment of acute exacerbations of chronic bronchitis", while other BNF mucolytics (carbocisteine, mecysteine and $\mathrm{N}$-acetylcysteine) have had more general or non-respiratory indications, e.g. "reduction in sputum viscosity". It is theorised that there is clinical evidence that erdosteine is useful in treatment of acute exacerbations of chronic bronchitis, unlike any of the other aforementioned mucolytics.

Methods In this narrative systematic review, databases utilised included: Medline, Embase and PubMed. Studies for inclusion had to be Randomised Controlled Trials (RCTs) primarily investigating the effect of erdosteine in exacerbations of chronic bronchitis or COPD. For comparison, RCTs were also included if they investigated carbocisteine, mecysteine and N-acetylcysteine's effects in exacerbations. A secondary outcome was to investigate the use of these mucolytics in improving COPD signs and symptoms.

Once selected, a two-stage publication elimination process was devised by the author to assess the quality of the trials.

Results Very few trials of adequate quality assessed the efficacy of mucolytics in chronic bronchitis or COPD. Of the 5,560 search results, only 62 trials investigated the aforementioned mucolytics use in chronic bronchitis or COPD, 41 were RCTs. Of the 41 RCTs only 13 were found to be of adequate quality; erdosteine (1 RCT), carbocisteine (3 RCTs), mecysteine (0 RCTs) and N-acetylcysteine (9 RCTs).

There was no evidence that erdosteine is useful in treating exacerbations, and very limited, weak evidence for some efficacy in exacerbation prevention. In contrast, carbocisteine showed some strong evidence of efficacy in preventing exacerbations, especially in Asian populations. $\mathrm{N}$-acetylcysteine trial results were variable, and evenly distributed between positive and no effects, with one study showing adverse effects. There were no trials of adequate quality investigating mecysteine.

Conclusion There is little evidence that erdosteine is useful in treating chronic bronchitis exacerbations, whereas, overall carbocisteine seems to be more efficacious in exacerbation prevention.

\section{P138 AN INNOVATIVE APPROACH TO STUDY DESIGN: USING ELECTRONIC MEDICAL RECORDS TO INFORM THE FEASIBILITY AND DESIGN OF THE NOVELTY STUDY (A NOVEL OBSERVATIONAL LONGITUDINAL STUDY ON PATIENTS WITH ASTHMA AND/OR COPD)}

${ }^{1} \mathrm{HK}$ Reddel, ${ }^{2} \mathrm{M}$ Gerhardsson de Verdier, ${ }^{3} \mathrm{~A}$ Agustí, ${ }^{4} \mathrm{R}$ Beasley, ${ }^{5} \mathrm{EH}$ Bel, ${ }^{6} \mathrm{C}$ Janson, ${ }^{7}$ B Make, ${ }^{7} \mathrm{RJ}$ Martin, ${ }^{8}$ I Pavord, ${ }^{9} \mathrm{D}$ Postma, ${ }^{10} \mathrm{D}$ Price, ${ }^{2} \mathrm{C}$ Keen, ${ }^{2} \mathrm{~A}$ Gardev, ${ }^{2} \mathrm{~S}$ Rennard, ${ }^{2} \mathrm{~A}$ Sveréus, ${ }^{11} \mathrm{AT}$ Bansal, ${ }^{2} \mathrm{~L}$ Brannman, ${ }^{2} \mathrm{~N}$ Karlsson, ${ }^{2} \mathrm{~J}$ Nuevo, ${ }^{2} \mathrm{~F}$ Nyberg, ${ }^{2} \mathrm{~S}$ Young, ${ }^{12} \mathrm{~J}$ Vestbo. 'Woolcock Institute of Medical Research, Sydney, Australia; ${ }^{2}$ AstraZeneca, Madrid, Spain; ${ }^{3}$ Hospital Clinic University of Barcelona, Barcelona, Spain; ${ }^{4}$ Medical Research Institute of New Zealand, Wellington, New Zealand; ${ }^{5}$ Academic Medical Centre, University of Amsterdam, Amsterdam, The Netherlands; ' Uppsala University, Uppsala, Sweden; ${ }^{7}$ National Jewish Health and University of Colorado, Denver, USA; ${ }^{8}$ University of Oxford, Oxford, UK; ${ }^{9}$ University of Groningen, University Medical Centre Groningen, Groningen, The Netherlands; ${ }^{10}$ Research in Real-Life, Singapore, Singapore; ${ }^{11}$ Acclarogen Ltd, Cambridge, UK; ${ }^{12}$ The University of Manchester, Manchester, UK

\subsection{6/thoraxjnl-2016-209333.281}

Introduction Asthma and COPD have traditionally been viewed as distinct diseases. While they have overlapping biological mechanisms, past studies often focused on specific aspects of each disease based on a single diagnostic label, and many clinical trial populations were defined by strict enrolment criteria with limited generalisability. NOVELTY is a prospective, multinational, observational, longitudinal cohort study aiming to enrol 14,800 patients aged $\geq 12$ years with a diagnosis or suspected diagnosis of asthma and/or COPD. In this population, the objectives of NOVELTY are to: (i) describe patient characteristics, treatment patterns and burden of illness over time in clinical practice; and (ii) use biomarkers and clinical parameters to identify phenotypes and endotypes associated with differential outcomes for symptom burden, clinical evolution and healthcare utilisation.

Aim This feasibility analysis of electronic medical records (EMRs) aimed to understand the potential study population, assess patient numbers across disease severities and evaluate EMRs as a data source for NOVELTY.

Methods EMRs from patients with asthma and/or COPD were identified from national databases covering primary and specialist care in 11 countries (Table). Disease severity was classified using treatment- and/or lung function-based algorithms for asthma and COPD. EMR variable coverage and completeness were assessed for standardised clinical, laboratory and physiological data and patient-reported outcomes (PROs).

Results EMRs for 921,888 patients with asthma, 958,945 with COPD and 117,893 with both diagnoses were identified. EMRs routinely documented patient demographics and characteristics, but many disease- and treatment-related data, and PROs/symptoms required for evaluation of disease severity and clinical outcomes were frequently missing (not collected or not documented; Table). Disease severity could not be classified in 561,837 patients (asthma) and 355,743 patients (COPD), representing $22-100 \%$ and $7-85 \%$ of patients across countries.

Conclusions EMR analysis revealed numbers of patients per country potentially eligible for NOVELTY. Many variables required to meet NOVELTY objectives were missing in EMRs (e. g. lung function and $\mathrm{PRO} /$ symptoms); therefore, variables in 
NOVELTY will primarily be documented in electronic case report forms, not EMRs. The lack of lung function data in many countries suggested divergences in diagnosis of asthma/COPD between clinical guidelines (which include lung function tests) and clinical practice.

\begin{tabular}{|c|c|c|c|c|c|c|}
\hline Country & $\begin{array}{l}\text { EMR } \\
\text { source }\end{array}$ & $\begin{array}{l}\text { Age/ } \\
\text { sex, \% }\end{array}$ & $\begin{array}{l}\text { Height/ } \\
\text { weight, \% }\end{array}$ & $\begin{array}{l}\text { Lung function } \\
\text { tests, \% }\end{array}$ & $\begin{array}{l}\text { Haemat-ology } \\
\text { tests, \% }\end{array}$ & $\begin{array}{l}\text { PROs, } \\
\%\end{array}$ \\
\hline Australia & A (GPs) & 100 & $21-32$ & 0 & $31-66$ & 0 \\
\hline Canada & A & 100 & $34-42$ & $1-10$ & $38-52$ & 0 \\
\hline China & $\begin{array}{l}\text { B } \\
\text { (outpatient) }\end{array}$ & 100 & 0 & 0 & $2-83$ & 0 \\
\hline France & A & 99-100 & $13-46$ & 0 & $7-19$ & 0 \\
\hline Germany & A & 100 & $18-23$ & 0 & 0 & 0 \\
\hline Italy & A & 79-100 & $18-24$ & $0-4$ & $13-23$ & 0 \\
\hline Japan & B & 100 & 0 & 0 & 0 & 0 \\
\hline Spain & A & 100 & $24-41$ & 0 & $26-35$ & 0 \\
\hline Sweden & c & 100 & $34-55$ & $33-56$ & NA & 0 \\
\hline UK & A (GPs) & 100 & $30-72$ & $0-57$ & $31-59$ & 0 \\
\hline USA & $A, C$ & 100 & $32-100$ & $0-17$ & $10->70$ & 0 \\
\hline
\end{tabular}

A, physician records; B, hospital records; C, registries; COPD, chronic obstructive pulmonary disease; EMR, electronic medical record; GP, general practitioner; NA, available but not assessed; PROs, patient-reported outcomes.

Please refer to page A271 for declarations of interest in relation to abstract P138.

\section{P139 THE BURDEN OF COPD ACROSS THE EUROPEAN UNION: DEVELOPMENT OF THE EUROPEAN COPD ATLAS}

${ }^{1} \mathrm{JFM}$ van Boven, ${ }^{1} \mathrm{~J}$ Gaughan, ${ }^{2} \mathrm{JB}$ Soriano, ${ }^{3} \mathrm{~J}$ Correia de Sousa, ${ }^{4} \mathrm{~N}$ Baxter, ${ }^{5} \mathrm{M}$ RománRodríguez, ${ }^{6} \mathrm{~J}$ Vilaró, ${ }^{7} \mathrm{~S}$ Williams, ${ }^{8} \mathrm{~S}$ Fitch, ${ }^{9} \mathrm{~K}$ Kishore, ${ }^{9} \mathrm{H}$ Chaudhury. ${ }^{1}$ European COPD Coalition, Brussels, Belgium; ${ }^{2}$ Instituto de Investigación Hospital Universitario de la Princesa (IISP), Universidad Autónoma de Madrid, Madrid, Spain; ' ${ }^{3}$ Life and Health Sciences Research Institute (ICVS), School of Health Sciences, University of Minho, Braga, Portugal, ICVS/3B's PT Government Associate Laboratory, Braga/Guimarães, Portugal; ${ }^{4}$ NHS Southwark Clinical Commissioning Group, London, UK; ${ }^{5}$ Instituto de Investigación Sanitaria de las Islas Baleres (IdISPa) Balearic Health Service, Palma de Mallorca, Spain; ${ }^{6}$ Department of Physiotherapy, Ramon Llull University, Barcelona, Spain; ${ }^{7}$ International Primary Care Respiratory Group, Westhill, UK; ${ }^{8}$ Fundación Lovexair, Madrid, Spain; ${ }^{9}$ Health IQ, London, UK

\subsection{6/thoraxjnl-2016-209333.282}

Background Estimating current and future impact of chronic obstructive pulmonary disease (COPD) within the European Union (EU) is essential for targeted and well-informed policymaking, however, current global and regional estimates are contradictory, and comparable standardised data is lacking. Without it the burden felt by individuals and healthcare systems cannot be fully quantified and a collective and coordinated response cannot be achieved to protect economies and communities from further harm.

Aim Our purpose was to

- Collect data to enable a comparative assessment of the COPD burden across EU 28 member states and highlight variation.

- Show the impact of COPD in the workplace, on healthcare utilisation and on quality of life of the EU population.

- Use the data to underpin a simulation model to demonstrate future impact on societies, health inequalities and healthcare utilisation depending on which interventions are selected.
Methods A systematic literature review was performed to identify regional and national data on COPD prevalence, risk factors (e.g. smoking, air pollution), impact and costs across the EU. Workshops with stakeholders from a range of European countries were convened to test the face validity of the data, and to develop policy-level questions from which a simulation model could be developed.

Results Prevalence estimates of COPD varied considerably $(1.26 \%$ to $13.87 \%)$, partly because of different definitions. As smoking rates are less affected by definition differences, these were used in a model that predicts COPD incidence, prevalence and mortality. Correction factors were applied to account for non-smoking related causes and under diagnosis of smoking. Smoking prevalence rates were used to estimate data for equivalent countries for the countries where smoking data were not available. The simulation model is currently in development and first results are expected in Autumn 2016.

Conclusion The European COPD Coalition (ECC) will use the results to facilitate dialogue with EU decision makers (European Commission Council and Parliament) on health policy, outlining the problem and providing evidence to support the call for political actions on COPD. It will also be of significant interest to healthcare professionals, patients, and respiratory organisations with a passion to improve COPD care

\section{P140 COMORBIDITIES OF SWEDISH PATIENTS DIAGNOSED WITH CHRONIC OBSTRUCTIVE PULMONARY DISEASE (COPD) AND/OR ASTHMA}

${ }^{1} \mathrm{G}$ Johansson, ${ }^{1} \mathrm{C}$ Jansen, ${ }^{2} \mathrm{M}$ Van Der Tol, ${ }^{3} \mathrm{R}$ Ariely, ${ }^{4} \mathrm{G}$ Bergman, ${ }^{4} \mathrm{M}$ Unde, ${ }^{4} \mathrm{P}$ Sobocki, ${ }^{2} \mathrm{H}$ Benhaddi. ${ }^{1}$ Uppsala University, Uppsala, Sweden; ${ }^{2}$ Teva Pharmaceuticals (Amsterdam), Amsterdam, The Netherlands; ${ }^{3}$ Teva Pharmaceuticals (Frazer), Frazer, USA; ${ }^{4}$ MS Health, Solma, Sweden

10.1136/thoraxjnl-2016-209333.283

Introduction and objectives Standard of care treatments for asthma and COPD are commonly administered in single-dose or multidose dry powder inhalers. There is a dearth of evidence around the prevalence of comorbidities, especially those that may affect inhaler device handling, among Swedish asthma and COPD patients.

Methods This retrospective study from the Swedish National Health Registries included 495,254 patients receiving inpatient or specialised outpatient care in Sweden between January 1, 2005 and December 31, 2014. Estimates of severity were based on number of asthma/COPD drugs used. Diagnostic codes were used to assess number of patients with a pre-specified comorbidity potentially affecting device handling.

Results Patient characteristics, treatments and comorbidities are summarised in the Table. Comorbidities that may impact inhaler handling were observed in 15.8\% (asthma), 50.4\% (COPD) and $55.3 \%$ (asthma/COPD) patients; incidence was increased with disease severity (patients with severe disease: 26.3\%, 52.0\%, $55.9 \%$ ) and advanced age (patients 60-69 years: 33.2\%, 45.2\%, $50.5 \%$, respectively).

Conclusions Comorbidities potentially affecting device handling were common across all groups, and unexpectedly high among elderly asthma patients. Furthermore, the data indicate that a substantial percentage of patients use two or more separate inhalers. These findings highlight the need for newer, easier to use inhalers, as well as training and monitoring of device use in patients who may have more difficulties using their devices correctly due to comorbidities. 


\section{Correction}

Reddel HK, Gerhardsson de Verdier M, Agustí A, et al. P138 An innovative approach to study design: using electronic medical records to inform the feasibility and design of the novelty study (a novel observational longitudinal study on patients with asthma and/or COPD). Thorax 2016;71:A157-A158. doi:10.1136/thoraxjnl-2016-209333.281

The affiliations for some authors has been corrected. The changes are indication in bold:

HK Reddel ${ }^{1}$, M Gerhardsson de Verdier $^{2}$, A Agustí ${ }^{3}$, R Beasley ${ }^{4}$, EH Bel ${ }^{5}, \mathrm{C} \mathrm{Janson}^{6}$, B Make ${ }^{7}$, RJ Martin ${ }^{7}$, I Pavord ${ }^{8}$, D Postma ${ }^{9}$, D Price ${ }^{10}, \mathrm{C} \mathrm{Keen}^{2}$, A Gardev $^{11}$, S Rennard ${ }^{11}$, A Sveréus ${ }^{2}$, AT Bansal ${ }^{12}$, L Brannman ${ }^{13}, \mathrm{~N} \mathrm{Karlsson}^{2}, \mathrm{~J} \mathrm{Nuevo}^{14}$, F Nyberg ${ }^{2}, \mathrm{~S}$ Young ${ }^{11}$, J Vestbo ${ }^{15}$

${ }^{1}$ Woolcock Institute of Medical Research, Sydney, Australia

${ }^{2}$ AstraZeneca, Madrid, Spain

${ }^{3}$ Hospital Clinic University of Barcelona, Barcelona, Spain

${ }^{4}$ Medical Research Institute of New Zealand, Wellington, New Zealand

${ }^{5}$ Academic Medical Centre, University of Amsterdam, Amsterdam, The Netherlands

${ }^{6}$ Uppsala University, Uppsala, Sweden

${ }^{7}$ National Jewish Health and University of Colorado, Denver, USA

${ }^{8}$ University of Oxford, Oxford, UK

${ }^{9}$ University of Groningen, University Medical Centre Groningen, Groningen, The Netherlands

${ }^{10}$ Research in Real-Life, Singapore, Singapore

${ }^{11}$ AstraZeneca, Cambridge, UK

${ }^{12}$ Acclarogen Ltd, Cambridge, UK

${ }^{13}$ AstraZeneca, Gaithersburg, MD, USA

${ }^{14}$ AstraZeneca, Madrid, Spain

${ }^{15}$ The University of Manchester, Manchester, UK

Thorax 2017;72:235. doi:10.1136/thoraxjnl-2016-209333.281corr1

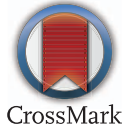

